\title{
Introdução à Reprodutibilidade de Experimentos Computacionais de Alto Desempenho
}

\author{
Vinícius Garcia Pinto, Lucas Leandro Nesi, Lucas Mello Schnorr \\ Instituto de Informática, Universidade Federal do Rio Grande do Sul (UFRGS) \\ Caixa Postal 15.064 - 91.501-970 - Porto Alegre - RS - Brasil \\ \{vgpinto, llnesi, schnorr\}einf.ufrgs.br
}

\begin{abstract}
Resumo. Este tutorial introduz uma metodologia reprodutível para realização de experimentos na área de processamento paralelo de alto desempenho. $O$ objetivo é motivar a adoção de boas práticas experimentais que possibilitem a coleta de medidas mais representativas e que por consequência levem a resultados mais confiáveis. A metologia apresentada é organizada em duas fases: execução de experimentos e análise dos dados. São sugeridas técnicas e ferramentas apropriadas para cada uma das etapas que compõe estas duas fases.
\end{abstract}

\section{Introdução}

Reprodutibilidade é um aspecto chave da pesquisa científica que possibilita que observações e experimentos possam ser refeitos de maneira independente. No área de processamento de alto desempenho, é natural lidar com múltiplos computadores que por vezes também são especializados, ou seja, contêm placas aceleradoras e interfaces de rede com grande largura de banda e baixa latência.

Experimentos computacionais confiáveis precisam envolver a coleta de medidas representativas. Isso implica registrar e, quando possível, controlar a configuração de hardware e software usada nos testes. Idealmente cada resultado produzido deve ser acompanhado do respectivo registro da plataforma, permitindo identificar e correlacionar aqueles aspectos que possam influenciar o experimento em questão, tais como atualizações de software ou melhorias no hardware. Manter esse fluxo de trabalho torna os experimentos naturalmente mais rigorosos, exigindo maior atenção. Este esforço pode ser diluído por meio da criação de scripts executados automaticamente no momento da execução de cada experimento. Tal disciplina estende-se também ao posterior trabalho nos dados coletados como obtenção de medidas de desempenho, tais como médias, mínimos, máximos, dispersão e na elaboração de gráficos e tabelas.

Este minicurso aborda uma introdução à reprodutibilidade de experimentos computacionais de alto desempenho. Apresentamos as técnicas e conceitos com o objetivo de motivar a adoção de procedimentos disciplinados, que levem a conclusões que não apenas evidenciem os efeitos observados mas que sejam portáveis no tempo (futuras observações) e no espaço (outras plataformas computacionais semelhantes). Uma abordagem aprofundada é discutida em [Pinto et al. 2020]; materiais e referências complementares são catalogados em: https://exp-hpc.gitlab.io/

\section{Visão Geral da Metodologia}

A metodologia para experimentos reprodutíveis se inspira no trabalho de Jain [Jain 1991]. A Figura 1 ilustra a sua organização em duas fases. A partir dos objetivos estabelecidos 
para investigação, define-se o projeto experimental com fatores (de controle) e variáveis de resposta. A execução dos projeto experimental (esquerda da linha pontilhada) envolve também a coleta automatizada de informações da plataforma e da aplicação por meio de scripts. O projeto combina, replica e torna aleatória a ordem de execução para reduzir o impacto do indeterminismo de anomalias durante os experimentos. Ao final dos experimentos, os dados coletados compreendem tanto informações constantes da aplicação e da plataforma registrados, assim como dados de entrada e de saída da execução.

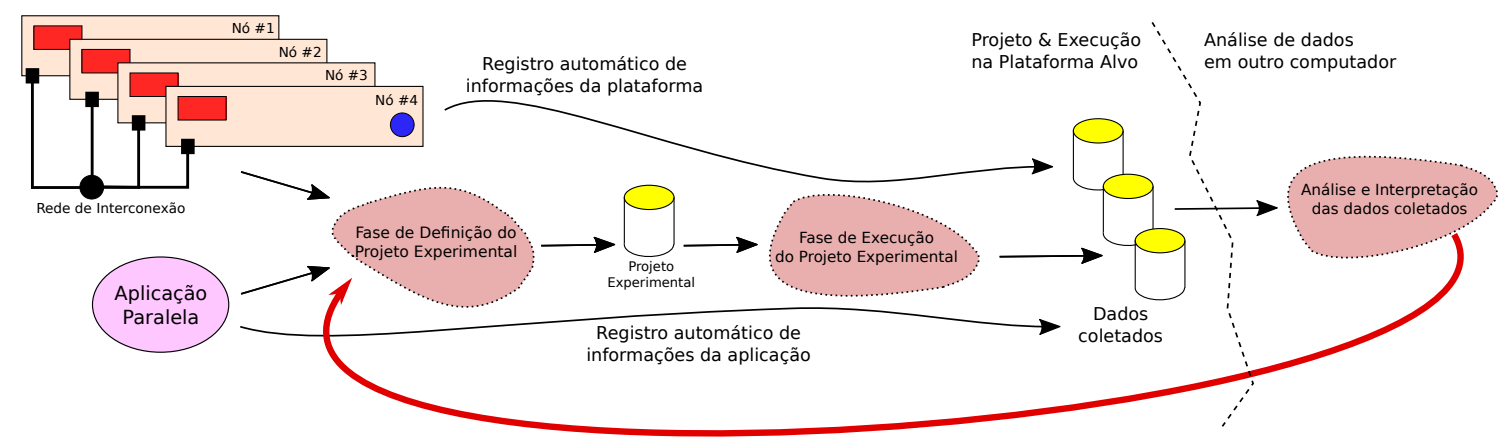

Figura 1. Metologia experimental com enfoque na reprodutibilidade.

A análise dos dados (direita da linha pontilhada na 1) coletados é realizada após a execução da bateria experimental. É um processo que se inicia com uma análise geral dos resultados observados ao qual se segue um aprofundamento em pontos específicos. É comum que tais aprofundamentos revelem novos pontos a serem investigados e que, por consequência, novas baterias experimentais surjam a partir de observações de experimentos anteriores, fazendo da análise um processo cíclico. Cabe ressaltar que a automatização por scripts não desaparece totalmente, devendo ser aplicada nas etapas de transformação dos dados brutos e na geração de gráficos. Idealmente, tal código deve acompanhar as reflexões permitindo que se possa revisitar o fluxo de ideias que levou a uma dada conclusão.

\section{Implementação da Metodologia}

A metodologia descrita requer a coleta de informações da plataforma e da aplicação, o gerenciamento da pilha de software e o controle da plataforma. Várias ferramentas e configurações já existem para realizar todos estes processos. Nós propomos listas (não exaustivas) para elas. Todas as ferramentas estão catalogadas e referenciadas no nosso companion 1]. Para a coleta de informações da plataforma e da aplicação sugerimos as seguintes ferramentas: Istopo (do pacote $h w l o c$ ) que mostra a topologia de hardware do sistema. cpufreq-info (cpufrequtils) que mostra informações como frequência dos processadores. nvidia-smi um comando equivalente para aceleradores NVIDIA. Ispci (Unix) que apresenta informações dos dispositivos PCI. ip, ifconfig, ou outra ferramenta dependendo do gerenciador de rede, para informações sobre as interconexões de rede.

Devido a complexidade da pilha de software das aplicações, sugerimos ferramentas para a instalação e controle de dependências. Um exemplo é a ferramenta spack [Gamblin et al. 2015]. Para obter informações da aplicação os seguintes comandos podem ser úteis. Idd para listar as bibliotecas compartilhadas utilizadas. env para mostrar

1 https://gitlab.com/exp-hpc/boas-praticas 
as variáveis de ambiente do sistema. nm para listar os símbolos da aplicação. ompi-info para mostrar as informações do middleware OpenMPI.

A execução dos experimentos é vastamente influenciada pela plataforma computacional utilizada e suas configurações. Para diminuir e controlar a variabilidade causada pela plataforma sugerimos: (i) Controle da Frequência dos processadores e aceleradores. (ii) Vinculação (Binding) dos fluxos de execução do programa (threads) nas unidades de processamento. (iii) Desativar recursos de hardware que podem causar variabilidade como Intel Turboboost, AMD Turbo Core e Simultaneous Multithreading (Intel HyperThreading). (iv) Considerar o fator NUMA, configurando as threads e dados conforme a topologia. (v) Configurar a interface de interconexão, como por exemplo as configurações padrões de TCP/IP. (vi) Configurações do Kernel, como Address space layout randomization (ASLR), escalonadores e prioridades dos processos, e driver dos recursos.

A metodologia sugere a utilização da filosofia literate programming [Knuth 1984] que faz o uso de journals e notebooks. Estes documentos contêm linguagem natural descrevendo as atividades, blocos de código para a programação, além de dados e imagens para as análises de dados. Ambientes como emacs + ess + org-mode, RStudio, e Jupyter Notebook são algumas opções. A etapa de data science pode fazer o uso de ferramentas consagradas como a linguagem de programação $\mathbf{R}$, com a biblioteca tidyverse, a linguagem de programação Python, com a biblioteca Pandas, ou a linguagem de programação Julia. Para a criação de design de experimentos os seguintes pacotes/bibliotecas podem ser utilizados: DoE.base (R), FrF2 (R), pyDOE2 (Python) e ExperimentalDesign (Julia).

\section{Conclusão}

Este texto apresenta uma breve introdução à reprodutibilidade de experimentos computacionais com objetivo de motivar o emprego de boas práticas pelo público de processamento paralelo de alto desempenho. A metodologia discutida abrange tanto a etapa de projeto e coleta de resultados experimentais quanto a posterior análise dos mesmos. Para cada etapa destes processos, são listados, a título de exemplo, algumas sugestões de procedimentos e ferramentas que podem ser usados para implementação da metodologia.

Uma vez que experimentos diferentes possuem requisitos e particularidades distintas, alguns dos pontos aqui discutidos podem ser insuficientes ou inaplicáveis. Esperase entretanto que o presente material sirva de motivação e como ponto de partida para adoção de práticas que levem a resultados mais confiáveis.

\section{Agradecimentos}

Este trabalho foi realizado com o apoio da Coordenação de Aperfeiçoamento de Pessoal de Nível Superior (CAPES) - Finance Code 001, do Conselho Nacional de Desenvolvimento Científico e Tecnológico (CNPq) com a bolsa para o $2^{\circ}$ autor (141971/20207), e dos projetos: FAPERGS ReDaS (19/711-6), MultiGPU (16/354-8) e GreenCloud (16/488-9), do projeto CNPq 447311/2014-0, do projeto CAPES/Brafitec 182/15 e CAPES/Cofecub 899/18, e com apoio do projeto Petrobras (2018/00263-5). 


\section{Referências}

Gamblin, T., LeGendre, M., Collette, M. R., Lee, G. L., Moody, A., de Supinski, B. R., and Futral, S. (2015). The spack package manager: Bringing order to hpc software chaos. In High Performance Computing, Networking, Storage and Analysis, 2015 SCInternational Conference for, pages 1-12. IEEE.

Jain, R. (1991). The Art of Computer Systems Performance Analysis: Techniques for Experimental Design, Measurement, Simulation, and Modeling. Wiley.

Knuth, D. E. (1984). Literate Programming. The Computer Journal, 27(2):97-111.

Pinto, V., Nesi, L., and Schnorr, L. (2020). Boas Práticas para Experimentos Computacionais de Alto Desempenho. In du Bois, A. and Castro, M., editors, Minicursos da XX Escola Regional de Alto Desempenho da Região Sul, chapter 1, pages 1-19. SBC, Porto Alegre. 